\title{
Clinical application of cerebrospinal fluid cytology in China
}

\author{
Hong-Zhi Guan', Gang Zhao \\ 'Department of Neurology, Peking Union Medical College Hospital, Chinese Academy of Medical Sciences, Beijing 100730, China. \\ ${ }^{2}$ Department of Neurology, Xijing Hospital, the Fourth Military Medical University, Xi'an 710032, Shaanxi, China. \\ Correspondence to: Prof. Hong-Zhi Guan, Department of Neurology, Peking Union Meidical College Hospital, Chinese Academy of \\ Medical Sciences, Beijing 100730, China. E-mail: guanhz@263.net; Prof. Gang Zhao, Department of Neurology, Xijing Hospital, the \\ Fourth Military Medical University, Xi'an 710032, Shaanxi, China. E-mail: zhaogang@fmmu.edu.cn
}

\begin{abstract}
How to cite this article: Guan HZ, Zhao G. Clinical application of cerebrospinal fluid cytology in China. Neuroimmunol Neuroinflammation 2018;5:4. http://dx.doi.org/10.20517/2347-8659.2017.52
\end{abstract}

Received: 17 Oct 2017 Frist Decision: 15 Jan 2018 Revised: 26 Jan 2018 Accepted: 29 Jan 2018 Published: 11 Feb 2018

Science Editor: Athanassios P. Kyritsis Copy Editor: Jun-Yao Li Production Editor: Huan-Liang Wu

\begin{abstract}
Cerebrospinal fluid (CSF) cytology is a fundamental test for the diagnosis of central nervous system (CNS) disease in neurological practice in China. CSF cytology provides diagnostic evidence in cases suspected of CNS infectious diseases, autoimmune encephalitis and CNS neoplastic disorders. Immunocytochemistry and flow cytometry make the cytological diagnosis more sensitive and specific. However, the diagnostic significance of CSF cytology should be interpreted and evaluated in the context of specific clinical backgrounds. Although molecular diagnostic techniques, including polymerase chain reaction and next-generation sequencing, are increasingly applied to CSF examination, the classical CSF cytology still has its horizon in the era of precision neurology and should be recommended as a routine test for CSF analysis.
\end{abstract}

Keywords: Cerebrospinal fluid, cytology, neurology, infection, autoimmune

\section{INTRODUCTION}

Analysis of cerebrospinal fluid (CSF) became possible with the introduction of lumbar puncture (LP) for clinical application at the beginning of the 20th century. Initial reports in the diagnostic utility of LP mainly focused on diagnosis of central nervous system (CNS) infectious disease, particularly neurosyphilis. Two reports published in 1904 describing findings of CNS tumors in CSF were seen as the beginning of CSF cytology. One was by Dufour, a French physician; the other by Walter Rindfleisch ${ }^{[1]}$, a German physician. However the CSF cytology wasn't fully developed until the invention of CSF concentration techniques in

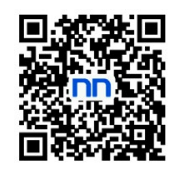



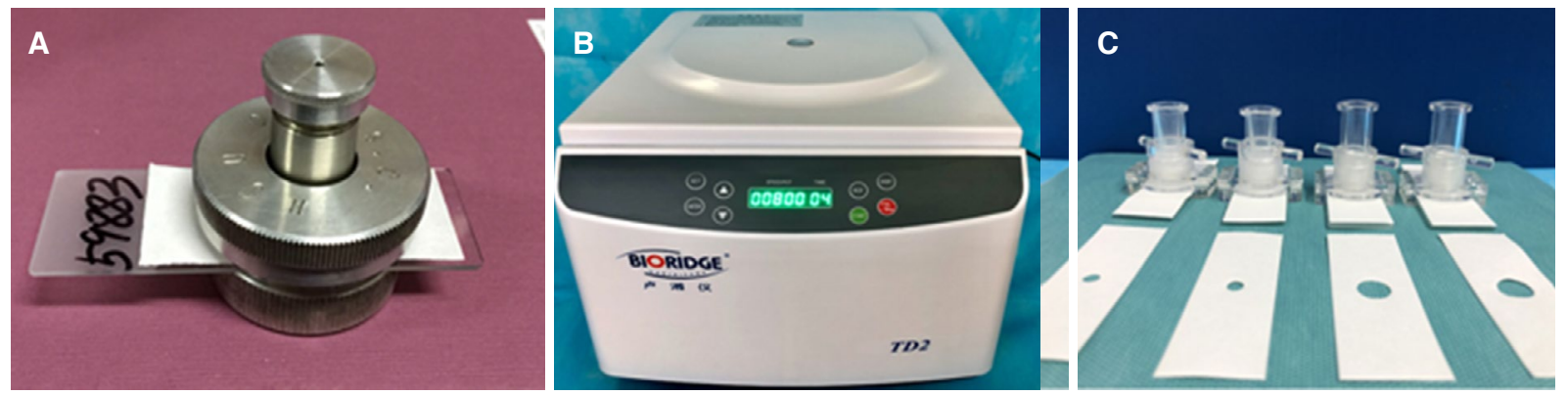

Figure 1. (A) The Hou's chamber modified from the Sayk's method which is still in use in some CSF labs in China; (B) the cytocentrifuge equipment for the Su's Centrifugal method (photo provided by Yi-Ning Yang from Xijing Hospital); (C) the chambers used in the Su's Centrifugal method (photo provided by Yi-Ning Yang)

the 1950 s by the neurologist Sayk ${ }^{[2]}$ (1923-2005), a neurologist from East Germany. He developed Sayk's cell sedimentation chamber which was modified later and is still used in some labs in China.

CSF cytology in China mainland dates back to the 1960 s and 1970s when famous Chinese neurologists, including Hou et al. ${ }^{[3]}$, Su et al. ${ }^{[4]}$, introduced the technique to major neurological centers in China. These pioneers' contribution laid the foundation of clinical CSF research in China mainland. The CSF Cytology Society was founded in 1991 as a division of the Chinese Society of Neurology, which later was renamed as the Neuroinfection and CSF Cytology Society under suggestions of its current chairman, Dr. Gang Zhao. Currently, many neurologists read slides and sign CSF cytology reports under the certification of the Chinese Society of Neurology. The historic background of CSF cytology in China made CSF cytology a wellaccepted diagnostic method in our neurological practice ${ }^{[5]}$.

A series of conferences and educational activities was organized by the society to ensure the diagnostic quality of different CSF cytology labs around China. Neurologists in China also modified techniques for CSF cytology based on Sayk's method and the cytocentrifuge technique. In the CSF cytology lab of the Department of Neurology in Peking Union Medical College Hospital (PUMCH), slides are prepared with the cell sedimentation technique with the Hou's cell sedimentation chamber modified from the Sayk's method of spontaneous cell sedimentation technique [Figure 1]. We put $0.5 \mathrm{~mL}$ cerebrospinal fluid into the chamber and store overnight at $4{ }^{\circ} \mathrm{C}$, allowing the cells to precipitate onto the slides. The slides are then air-dried and stained with May-Grunwald-Giemsa stain (MGG) and immunocytochemistry. Chinese ink stain (also called India ink stain) is used if Cryptococcus is suspected on MGG stain. Presently, over 8000 samples of CSF cytology are handled annually with this technique in the Department of Neurology in PUMCH. In contrast, in the CSF cytology lab of the Department of Neurology in Xijing Hospital, slides are prepared with the cytocentrifuge technique with a series of equipment developed and modified by Dr. Xiu-Chu Su and his colleagues. Nearly 10,000 samples of CSF cytology are handled in the Xijing Hospital annually with various type of staining methods, including modified modified Ziehl-Neelsen stain ${ }^{[6]}$. The modified Ziehl-Neelsen stain, introduced to CSF cytology by Dr. Gang Zhao and his team, is more sensitive than the conventional Ziehl-Neelsen stain and is available in many CSF cytology labs in China. Finally, in the Department of Neurology in the Second Hospital of Hebei Medical University, Dr. Jun-Ying He and her team are now focusing their research on the diagnosis and treatment of meningeal malignancy and around 5000 samples of CSF cytology are handled annually in their lab.

Based on various preparation and staining techniques, CSF cytology has become a fundamental test for the diagnosis of CNS disease in neurological practice in China. CSF cytology is a quantitative or semiquantitative cytology method, which also emphasizes inflammatory findings and differential diagnoses (infectious or non-infectious etiology) beyond that of malignancy identification. 

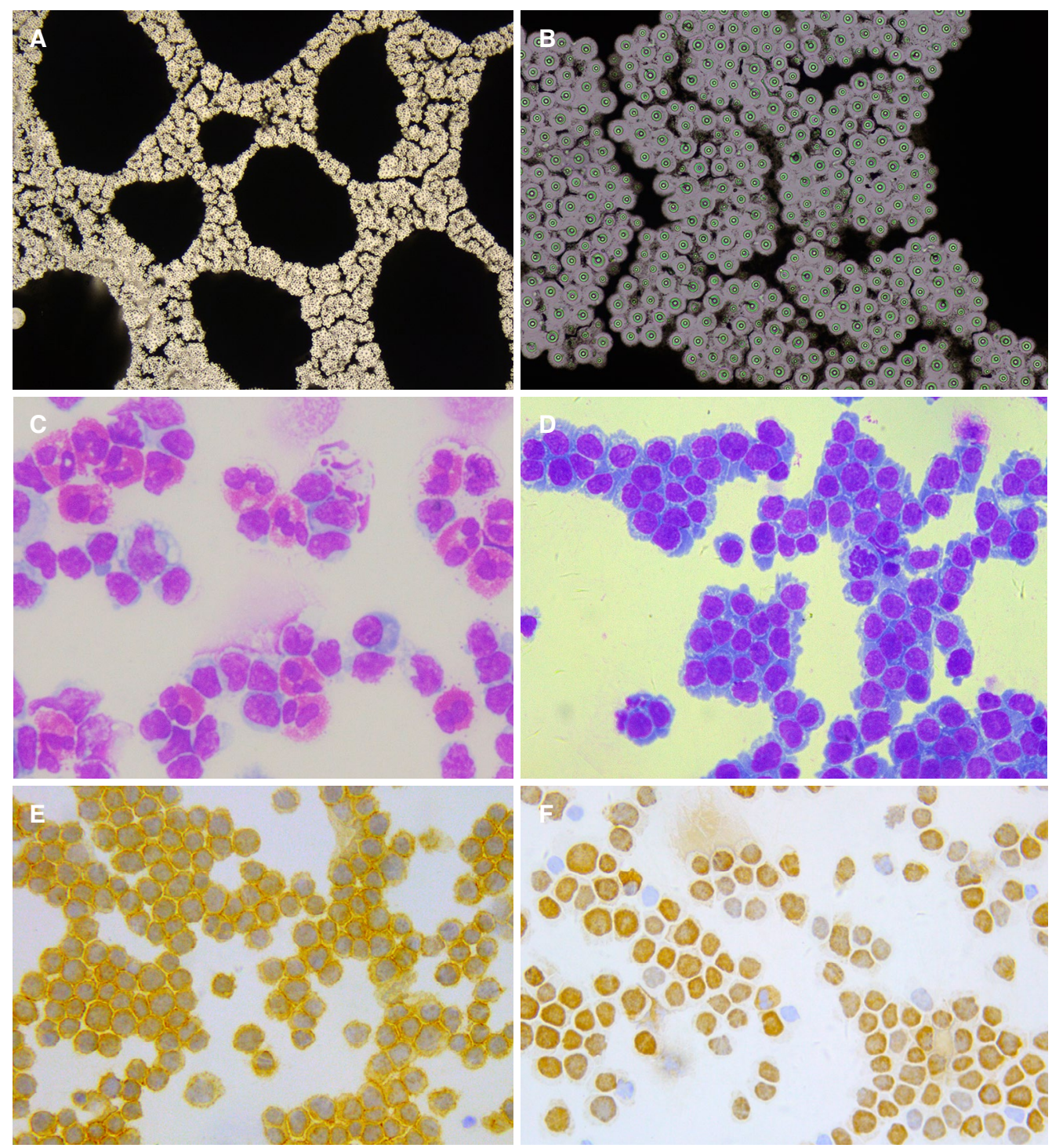

Figure 2. CSF cytology prepared with cell sedimentation chamber. (A) Cryptococcusgatti with Chinese ink staining, x100; (B) Cryptococcusgatti with Chinese ink staining, $x 400 ;$ (C) eosinophil pleocytosis in CSF of a patient with Angiostrongy/us cantonesis, MGG staining, x200; (D) lymphoma cells in CSF from a patient with PCNSL, MGG staining, $x 200$; (E) CD20 positive lymphoma cells in CSF, immunocytochemistry, x200; (F) Ki-67 index: 60\%, immunocytochemistry, x200. CSF: cerebrospinal fluid; MGG: May-GrunwaldGiemsa; PCNSL: primary central nervous system lymphoma

\section{CSF CYTOLOGY OF NEUROINFECTIOUS DISEASE}

Inflammatory disorders, either infectious or autoimmune diseases of the CNS, usually induce significant changes in the cellular content of the CSF.

Types of infection involving CNS include bacterial, viral, mycotic (fungi), parasite and prion infections. Specific infectious organisms identified by CSF cytology include Cryptococcus, Toxoplasma and various species of bacteriac. Cryptococcus is the most common infectious agent observed by CSF cytology [Figure 2]; however, this kind of specific pathogenic findings only account for a small percentage of all cytology diagnoses.

Nearly all types of CNS infections (except prion disease) have CSF cytological changes that may provide diagnostic clues of etiology. Acute bacterial infections usually induce neutrophil pleocytosis in CSF. Later on these infections also show lymphocytic pleocytosis. Acute tuberculous meningitis may also be characterized 
by neutrophil pleocytosis or a mixed cellular response with neutrophils and lymphocytes. In later stages of tuberculous meningitis, lymphocytic inflammation predominates while neutrophils remain present until ful clinical remission. The cytological picture of CNS viral infection predominantly shows a lymphocytic response. However, neutrophil pleocytosis may present in necrotizing cerebral processes of acute viral infections (e.g. herpes simplex virus-1 encephalitis or CNS vasculitis). Eosinophilic pleocytosis in the CSF is a hint at a parasitic infection, such as neurocysticercosis, a relatively common type of parasitic infection in North China. A cytological picture of eosinophil predominance in CSF, so called "eosinophilic meningitis", indicates either a special type of neurocysticercosis (e.g. meningeal type of cysticercosis) or Angiostrongylus cantonesis, mostly in Southeastern China ${ }^{[7]}$ [Figure 2].

The presence of plasma cells, the antibody-synthetic cells transformed from B-lymphocyte, in CSF indicates a chronic infection (e.g. tuberculosis, syphilis or borreliosis) or intrathecal humoral immunological response. Some infections lead to a higher content of atypical lymphoid or lymphoblastic cells in the CSF, which makes the differential diagnosis from lymphoma very difficult.

\section{CSF CYTOLOGY OF AUTOIMMUNE CNS DISEASE}

Autoimmune CNS disorders include primary neuroimmune disorders [e.g. multiple sclerosis (MS), neuromyelitis optica spectrum disorders (NMOSD) and autoimmune encephalitis] and those secondary to systemic autoimmune disease.

Inflammatory reactions in CSF are observed in about half of AQP4-IgG positive NMOSD patients. The main inflammatory type is lymphocytic inflammation, while neutrophils and eosinophils are also seen in a few patients. Activated lymphocytes, activated mononuclear cells, and plasma cells can be detected in some patients' CSF. The positive rate of the specific oligoclonal bands in AQP4-IgG positive NMOSD is lower than that in multiple sclerosis. These CSF characteristics may contribute to the diagnosis of NMOSD. A mildly increased percentage of eosinophils is common in autoimmune CNS demyelination including NMOSD, acute disseminated encephalomyelitis and transverse myelitis. The demonstration of plasma cells in CSF of patients with MS is significantly correlated with pleocytosis and intrathecal IgG synthesis ${ }^{[8]}$.

Anti-NMDAR encephalitis is a major type of autoimmune encephalitis associated with autoantibodies against neuronal surface proteins. Intrathecal synthesis of autoantibodies is a pathological mechanism of this disease. CSF cytology findings include lymphacytic inflammation, occasionally with a mild increased percentage of neutrophils. Plasma cells are common in CSF of anti-NMDAR encephalitis which indicates intrathecal immunoglobulin synthesis ${ }^{[9]}$. For other type of autoimmune encephalitis without the mechanism of intrathecal immunoglobulin synthesis (e.g. anti-LGI1 encephalitis and anti-GABAbR encephalitis), the CSF cytology findings are normal or less reactive ${ }^{[10]}$.

Neuro-Behçet Syndrome (NBS) may present with various clinical manifestations. The most common neuroimaging finding is CNS midline structure involvement including brainstem, basal ganglion and thalamus. The CSF cytological findings, typically the presence of neutrophils, indicate the nature of neutrophilic vasculitic of NBS and are important clues in the diagnosis of NBS.

Neuropsychiatric systemic lupus erythematosus (SLE) is the CNS involvement of SLE. According to our observation, he CSF cytology was abnormal in $32.9 \%$ of SLE patients, which showed lymphocytic inflammatory reactions or lymphocyte - neutrophil inflammation. Among these cases with positive CSF cytological findings, activated lymphocytes, plasma cells and activated monocytes were frequently present. Abnormal endocytosis of monocytes, which presented as monocytes phagocytosing lymphocytes or plasma cells, was shown in some cases ${ }^{[11]}$. 
Table 1. CSF cytological syndrome with etiological indication

\begin{tabular}{|c|c|c|}
\hline CSF cytology & Clinical syndrome & Etiological consideration \\
\hline Lymphatic inflammation & Acute encephalitis & $\begin{array}{l}\text { Viral encephalitis } \\
\text { Autoimmune encephalitis }\end{array}$ \\
\hline Lymphatic inflammation & Acute mild or moderated meningism & Viral meningitis \\
\hline Lymphatic inflammation & $\begin{array}{l}\text { Shepherd with chronic meningitis or } \\
\text { chronic encephalomyelitis }\end{array}$ & Neurobucellosis \\
\hline Mixed reaction with lymphocyte and neutrophil & $\begin{array}{l}\text { Recurrent brain-stem encephalitis or } \\
\text { diencephalitis }\end{array}$ & Neuro-Behçet disease \\
\hline Mixed reaction with lymphocytes and neutrophils & Chronic or subacute encephalitis & $\begin{array}{l}\text { Tubercular meningitis } \\
\text { Cryptococcal meningitis }\end{array}$ \\
\hline $\begin{array}{l}\text { Mixed reaction with Lymphocytes, eosinophils and } \\
\text { plasma cells }\end{array}$ & Chronic meningitis & Neurocysticercosis \\
\hline Eosinophilic inflammatory & $\begin{array}{l}\text { Acute meningitis in patients from } \\
\text { Southeastern China }\end{array}$ & Angiostrongylus cantonesis \\
\hline $\begin{array}{l}\text { Lymphatic inflammation with mild increased } \\
\text { eosinophils }\end{array}$ & Acute encephalomyelitis or myelitis & $\begin{array}{l}\text { Transverse myelitis } \\
\text { ADEM } \\
\text { NMOSD }\end{array}$ \\
\hline
\end{tabular}

CSF: cerebrospinal fluid; NMDAR: N-methyl-D-aspartate receptor; ADEM: acute disseminated encephalomyelitis; NMOSD: neuromyelitis optica spectrum disorder

CSF cytology findings in CNS inflammatory disease are relatively non-specific. However, when we consider the cytological findings under the clinical background of an individual patient, then the so-called "clinicalCSF cytological syndrome" can lead to a specific diagnosis [Table 1]. For example, in patients with recurrent CNS midline structure involvement and neutrophil pleocytosis or a mixed cellular response of CSF cytology, Neuro-Behçet disease should be highly suspected ${ }^{[12]}$.

\section{CSF CYTOLOGY OF NEOPLASTIC DISORDERS}

Identification of tumor cells by CSF cytology is direct and specific evidence of leptomeningeal involvement by neoplasms. The appearance of malignant cells in CSF usually indicates generalized seeding of the leptomeninges by tumor cells. The prevalence of leptomeningeal involvement of different tumors is important to the cytologists and clinicians to make an accurate clinical-cytoloical conclusion. According to Prayson and Fischler ${ }^{[13]}$, the most commonly identified malignancy in CSF specimens in adults is metastatic neoplasms. Primary central nervous system neoplasms (e.g. medulloblastoma) account for a higher percentage of CSF specimens in the pediatric population than in the adult population. We reviewed CSF cytology results from PUMCH between 1984 and 2003, including 3922 specimens ${ }^{[14]}$. Forty-nine cases (1.25\%) were positive for malignant cells. Diagnoses included metastatic tumors (26 cases), metastatic lymphoma/ leukemia (7 cases), primary CNS neoplasms (10 cases) and malignant unclassified neoplasms (6 cases).

Cytology deals with single cells without histological architecture, so cytologists often face the challenge of arriving at a definitive final diagnosis. Immunocytochemistry and immunophenotyping by flow cytometry are helpful for CSF cytology ${ }^{[15]}$. For example, it was estimated that CSF cytology combined with immunocytochemistry could indicate diagnostic findings in $50 \%$ of cases with primary central nervous system lymphoma (PCNSL). Flow cytometric analysis (FCA) and polymerase chain reaction (PCR) of rearranged IgH and TCR genes of CSF are widely used in the diagnosis of PCNSL ${ }^{[16,17]}$. To study the diagnostic value of CSF cytology in the diagnosis of PCNSL, we retrospectively analyzed the data of 21 patients of PCNSL with positive CSF cytological findings ${ }^{[18]}$. Conventional CSF cytology, immunocytochemistry, flow cytometric analysis and PCR of rearranged IgH and TCR genes of CSF were performed. The clinical and neuroimaging types of 21 patients included meningeal type $(n=13)$, parenchymal type $(n=4)$, ependymal type $(n=3)$, and optic type $(n=1)$. The CSF of all the 21 patients had atypical lymphocytes, suggestive of lymphoma. Of the 20 cases in which immunocytochemistry was performed, 17 showed B-lymphocyte predominance, which was consistent with the diagnosis of B cell 
lymphoma. FCA of 7 cases showed a significant increase in the percentage of B cells in 5 patients, indicating B cell lymphoma, and NK/T-lymphocyte predominance in 1 case, indicating an NK/T lymphoma. On analysis of the IgH and TCR genes in CSF of 4 patients, IgH monoclonal was found in 3 cases and TCR monoclonal in 1 case [Figure 2]. In fact, PCNSL is now one of the most common primary CNS neoplasms identified by comprehensive CSF cytological studies.

The application of molecular diagnostic techniques, including polymerase chain reaction and nextgeneration sequencing in CSF studies is booming in an era of precision medicine ${ }^{[19,20]}$. These novel techniques may be used to diagnose neoplastic meningopathy without cytological evidence ${ }^{[1,20]}$. However a final diagnosis based only on molecular techniques without morphological or cytological evidence should be avoided in clinical practice in order to prevent risks of over-diagnosis and misdiagnosis. CSF cytology is still the cornerstone of neurological diagnosis. The classical CSF examinations, including CSF cytology, still has their horizon in the era of precision neurology.

\section{DECLARATIONS}

\section{Acknowledgments}

The authors thank Yi-Ning Yang, Guo-Dong Feng, Jun-Ying He, Hui Bu and Yue-Li Zou for their correspondence on the information of their CSF lab.

\section{Authors' contributions}

Both authors drafted the manuscript, read and approved the final manuscript.

\section{Financial support and sponsorship}

None.

\section{Conflicts of interest}

There are no conflicts of interest.

\section{Patient consent}

Not applicable.

\section{Ethics approval}

Not applicable.

\section{Copyright}

(c) The Author(s) 2018.

\section{REFERENCES}

1. Dufour H. Classics in cytology. VIII: diffuse meningeal sarcomatosis with invasion of the spinal cord and the spinal roots. Positive cytological results, especially of the cerebrospinal fluid. 1904. Diagn Cytopathol 1997;17:67-9.

2. Sayk J. The results of the recent cytological studies of cerebrospinal fluid with sedimentation chamber. Arztl Wochensch 1954;9:1042-6. (in German)

3. Hou XD, Zhou SR. Clinical cerebrospinal fluid cytology. Nanjing: Jiangsu Science and Technology Press; 1985. p. 1-120. (in Chinese)

4. Su XC, Kong FY. Cerebrospinal fluid cytology of neurological disease. Beijing: People's Military Medical Press; 2001. p. 1-100. (in Chinese)

5. He JY, Kong FY, Guo L. Dragnosis of clincal cerebrospinal fluid cytology. Shijiazhuang: Hebei Science and Technology Press; 2007. p. 1-150. (in Chinese)

6. Chen P, Shi M, Feng GD, Liu JY, Wang BJ, Shi XD, Ma L, Liu XD, Yang YN, Dai W, Liu TT, He Y, Li JG, Hao XK, Zhao G. A highly efficient Ziehl-Neelsen stain: identifying de novo intracellular Mycobacterium tuberculosis and improving detection of extracellular M. tuberculosis in cerebrospinal fluid. J Clin Microbiol 2012;50:1166-70.

7. Guan HZ, Chen L, Cui LY, Guo YP, Huang Y, Zhou Y, Wang JM, Ren HT. Eosinophilic meningitis caused by angiostrongylus cantonensis: a clinical and cerebrospinal fluid cytology report of 9 cases. Chin J Neurol 2010;43:268-72. (in Chinese) 
8. Confavreux C, Caudie C, Touraine F, Ventre JJ, Aimard G, Devic M. Plasma cells in cerebrospinal fluid and multiple sclerosis: diagnostic yield and clinicobiological correlations. Acta Neurol Scand 1986;74:432-8.

9. Wang R, Guan HZ, Ren HT, Wang W, Hong Z, Zhou D. CSF findings in patients with anti-N-methyl-D-aspartate receptor-encephalitis. Seizure 2015;29:137-42.

10. Guan HZ, Ren HT, Yang XZ, Lu Q, Peng B, Zhu YC, Shao XQ, Hu YQ, Zhou D, Cui LY. Limbic encephalitis associated with anti-gammaaminobutyric acid B receptor antibodies: a case series from China. Chin Med J (Engl) 2015;128:3023-8.

11. Zhao YH, Shao YQ, Ren HT, Qian M, Guan HZ, Chen L. Cerebrospinal fluid cytology studies of neuropsychiatric systemic lupus erythematosus. Chin J Contemporary Neurol Neurosurg 2013;13:132-5. (in Chinese)

12. Guan HZ, Chen L, Wu QJ, Wang JM, Zhu YC, Ren HT, Cui LY. A clinical and cerebrospinal fluid cytology study of neuro-behcet syndrome. Chin J Neuroimmunol Neurol 2012;19:1-4. (in Chinese)

13. Prayson RA, Fischler DF. Cerebrospinal fluid cytology: an 11-year experience with 5951 specimens. Arch Pathol Lab Med 1998;122:47-51.

14. Guan HZ, Wang CH, Guo YP, Chen L, Ren HT, Zhao YH. Specific findings in cerebrospinal fluid cytology. Chin J Neurol 2004;37:65-7. (in Chinese)

15. Ren H, Zou Y, Zhao Y, Li J, Han X, He J, Guan H. Cerebrospinal fluid cytological diagnosis in multiple myeloma with leptomeningeal involvement: a report of two cases. Diagn Cytopathol 2017;45:66-8.

16. Fischer L, Martus P, Weller M, Klasen HA, Rohden B, Roth A, Storek B, Hummel M, Nagele T, Thiel E, Korfel A. Meningeal dissemination in primary CNS lymphoma: prospective evaluation of 282 patients. Neurology 2008;71:1102-8.

17. Guan HZ, Chen L, Guo YP, Cui W, Ren HT, Zhao YC, Gao J, Wang CH. Leptomeningeal lymphoma: cytological study of cerebrospinal fluid. Chin J Neurol 2006;39:113-7. (in Chinese)

18. Guan HZ, Chen L, Liang ZY, Zhong DR, Li J, Cui W, Qian M, Ren HT, Ling Q, Wang X, Zhao YH, Guo YP. Role of cerebrospinal fluid cytology in diagnosis of primary central nervous system lymphoma. Med J PUMCH 2012;3:273-8. (in Chinese)

19. Cui JZ, He JY, Li Q, Li XQ, Gao RP, Bu H, Zou YL, Guo XS, Han WX, Zhao ZY, Li YY, Zheng MM, Liu YJ, Yan LT. Advancements in diagnosis and treatment of meningeal carcinomatosis in solid cancer. Neuroimmunol Neuroinflammation 2017;4:167-78.

20. Zou Y, He J, Guo L, Bu H, Liu Y. Prediction of cerebrospinal fluid parameters for tuberculous meningitis. Diagn Cytopathol 2015;43:701-4. 